\title{
Rotational Activity around an Obstacle in 2D Cardiac Tissue in Presence of Cellular Heterogeneity
}

\author{
Pavel Konovalov ${ }^{1}\left(\mathbb{D}\right.$, Daria Mangileva ${ }^{1,2}$, Arsenii Dokuchaev ${ }^{1}\left(\mathbb{D}\right.$, Olga Solovyova $^{1,2} \mathbb{C}$ \\ and Alexander V. Panfilov $2,3,4, *$ (ID \\ 1 Institute of Immunology and Physiology, Ural Branch of Russian Academy of Sciences, \\ 620049 Ekaterinburg, Russia; p.konovalov@iip.uran.ru (P.K.); d.mangileva@iip.uran.ru (D.M.); \\ a.dokuchaev@iip.uran.ru (A.D.); o.solovyova@iip.uran.ru (O.S.) \\ 2 Laboratory of Computational Biology and Medicine, Ural Federal University, 620075 Ekaterinburg, Russia \\ 3 Department of Physics and Astronomy, Ghent University, 9000 Ghent, Belgium \\ 4 World-Class Research Center "Digital Biodesign and Personalized Healthcare", Sechenov University, \\ 119146 Moscow, Russia \\ * Correspondence: Alexander.Panfilov@ugent.be
}

check for updates

Citation: Konovalov, P.; Mangileva, D.; Dokuchaev, A.; Solovyova, O.; Panfilov, A.V. Rotational Activity around an Obstacle in 2D Cardiac Tissue in Presence of Cellular Heterogeneity. Mathematics 2021, 9, 3090. https://doi.org/10.3390/ math9233090

Academic Editors: Yuri Vassilevski and Vitaly Volpert

Received: 18 October 2021

Accepted: 27 November 2021

Published: 30 November 2021

Publisher's Note: MDPI stays neutral with regard to jurisdictional claims in published maps and institutional affiliations.

Copyright: (c) 2021 by the authors. Licensee MDPI, Basel, Switzerland. This article is an open access article distributed under the terms and conditions of the Creative Commons Attribution (CC BY) license (https:/ / creativecommons.org/licenses/by/ $4.0 /)$.

\begin{abstract}
Waves of electrical excitation rotating around an obstacle is one of the important mechanisms of dangerous cardiac arrhythmias occurring in the heart damaged by a post-infarction scar. Such a scar is also surrounded by the region of heterogeneity called a gray zone. In this paper, we perform the first comprehensive numerical study of various regimes of wave rotation around an obstacle surrounded by a gray zone. We use the TP06 cellular ionic model for human cardiomyocytes and study how the period and the pattern of wave rotation depend on the radius of a circular obstacle and the width of a circular gray zone. Our main conclusions are the following. The wave rotation regimes can be subdivided into three main classes: (1) functional rotation, (2) scar rotation and the newly found (3) gray zone rotation regimes. In the scar rotation regime, the wave rotates around the obstacle, while in the gray zone regime, the wave rotates around the gray zone. As a result, the period of rotation is determined by the perimeter of the scar, or gray zone perimeter correspondingly. The transition from the scar to the gray rotation regimes can be determined from the minimal period principle, formulated in this paper. We have also observed additional regimes associated with two types of dynamical instabilities which may affect or not affect the period of rotation. The results of this study can help to identify the factors determining the period of arrhythmias in post-infarction patients.
\end{abstract}

Keywords: re-entry; myocardial heterogeneity; cardiac modeling; myocardial infarction; infarct border zone; gray zone

\section{Introduction}

Propagating non-linear waves occur in various types of excitable media of a physical [1], chemical [2,3] and biological nature [4,5]. These waves can also form vortices which organize spatial excitation patterns in the medium and their onset can have important consequences $[4,6,7]$. For example, rotating waves in the heart are the main mechanisms of dangerous cardiac arrhythmias [8,9] and the study of such regimes in the heart is an important area of research in cardiac electrophysiology. In some patients, arrhythmias may arise from myocardial infarction, i.e., a condition where due to poor blood supply, a region of the heart is damaged. As a result, electrical waves propagating through the heart break. The breaks can occur due to direct interaction of the propagating wave with a heterogeneity, or due to dynamical instabilities [10]. Such breaks can form vortices, which often rotate in/around the damaged area [11,12].

From a mathematical point of view, such regimes can be viewed as rotation of nonlinear waves around an obstacle [13]. This is one of the classical regimes studied in the 
theory of excitable media. Such regimes were described in cellular automata models as early as in 1946 [14]. Later they were studied in reaction-diffusion models of excitable media [15]. More recently, interest in such regimes, especially on rotation around an obstacle of small size, was initiated by the concept of the anchoring of vortices proposed by Davidenko and co-authors [7]. Such processes were also studied using theoretical approaches [16,17].

Unfortunately, the direct application of such studies to arrhythmias which occur around an infarcted area is difficult due to the following problem. A post-infarction region in the heart contains a compact scar region, which is similar to an inexcitable obstacle [18]. Such a compact scar is surrounded by a border zone, which has properties that differ from the rest of the normal cardiac tissue [19]. This zone is also called the gray zone (GZ) because of its intermediate signal intensity in a clinical MRI [20]. In a mathematical sense, this problem can be viewed as a wave rotation around an obstacle surrounded by a region of a certain width in which the parameters of equations describing cardiac cells are different from those at the rest of the tissue [13,21]. Such rotation regimes are poorly understood.

The main aim of this paper is to perform a generic numerical study of this problem. We use a generic $2 \mathrm{D}$ representation of ventricular tissue with an infarction region, which is represented by a circular obstacle with a gray zone around it, defined by only two parameters: obstacle radius and gray zone width. We assume that properties of cardiomyocytes in the gray zone are different from those in the rest of the tissue and study the rotation of waves in such 2D models. (The details about gray zone representation are given in Section 2.2). We vary the size of the obstacle and gray zone to reveal their effects on the pattern of wave rotation and its period, which is an important characteristic of cardiac arrhythmia. We show that depending on the parameters, the wave can rotate either around the compact scar, or other regimes occur where the wave rotates within the gray zone or on its border with the normal tissue. We investigate the factors which determine the switching between these regimes and propose a basic rule which can predict transitions in such a system: the principle of minimal period. In addition, we show that the dependency of the period on the size of the scar in the physiologically relevant range is determined by the slope of the conduction velocity restitution curve, and can be estimated from a simple equation derived in the paper. We also find an additional regime of dynamical instabilities, which occurs for small scars and requires regional tissue heterogeneity. Overall, we provide classification of excitation patterns and determine the main factors and principles which can affect the period of the wave rotation in a generic model of the infarction scar. It can potentially contribute to understanding the mechanisms and dynamics of cardiac arrhythmias which occur in the hearts after a myocardial infarction.

\section{Materials and Methods}

In general, our numerical procedure is organized as follows. We first setup a 2D model of normal cardiac tissue, which consists of two parts: models of cardiac cells represented by 18 variable TP06 models, and a spatial operator (generalized Laplacian), which describes cell connections (Section 2.1). Then, we add to this 2D model an infarction scar surrounded by the gray zone. The infarction scar is modeled via boundary conditions and the gray zone as a heterogeneity, i.e., the gray zone is a domain in which the parameters of the TP06 model are different form the parameters in normal tissue (Section 2.2). Finally, we impose a special initial procedure (so-called S1-S2 protocol) in which we initiate a wave rotating around the gray zone (Section 2.3). After the wave is initiated, we find the period of rotation and study the spatial excitation patterns (Section 3).

\subsection{Baseline Ventricular Myocardial Tissue Model. Numerical Approach and Software}

To describe the propagation of the excitation wave in the ventricular myocardial tissue from a human heart, we used a 2D monodomain approach [22] for isotropic medium:

$$
C_{m} \frac{\partial V}{\partial t}=D \nabla^{2} V-I_{\text {ion }}
$$


where $V$ is the transmembrane potential; $\mathrm{D}$ is a constant accounting for electrical call coupling. $C_{m}$ is the capacitance of the cell membrane. $I_{i o n}$ is the sum of all transmembrane ionic currents, described with a biophysically detailed cellular ionic TP06 model of human ventricular cardiomyocytes [23]:

$$
I_{i o n}=I_{N a}+I_{K 1}+I_{t o}+I_{K r}+I_{K s}+I_{C a L}+I_{N a C a}+I_{N a K}+I_{p C a}+I_{p K}+I_{b N a}+I_{b C a},
$$

where $I_{N a}$ is the $\mathrm{Na}^{+}$current, $I_{K 1}$ is the inward rectifier $\mathrm{K}^{+}$current, $I_{t o}$ is the transient outward current, $I_{K r}$ is the delayed rectifier current, $I_{K s}$ is the slow delayed rectifier current, $I_{\mathrm{CaL}}$ is the L-type $\mathrm{Ca}^{2+}$ current, $I_{\mathrm{NaK}}$ is the $\mathrm{Na}^{+} / \mathrm{Ca}^{2+}$ exchanger current, $I_{\mathrm{NaK}}$ is the $\mathrm{Na}^{+} / \mathrm{K}^{+}$ATPhase current, $I_{p C a}$ and $I_{p K}$ are plateau $\mathrm{Ca}^{2+}$ and $\mathrm{K}^{+}$currents, and $I_{b N a}$ and $\mathrm{I}_{b \mathrm{Ca}}$ are background $\mathrm{Na}^{+}$and $\mathrm{Ca}^{2+}$ currents. This cellular model provides a detailed description of voltage, ionic currents and intracellular ion concentrations and is based on a wide range of human electrophysiological data. The properties of all of these currents are fitted to their experimentally measured values, and their dynamics are fitted using additional differential equations. Each of the currents is typically directly proportional to the corresponding constant $G_{*}$, called a maximal conductivity, and depends on the values of time-dependent variable $V$ and certain gating variables. To create heterogeneity, we regionally modified values of $G_{*}$ for several currents (please see the next subsection).

Simulations were performed on grids of $500 \times 500$ elements $(140 \mathrm{~mm} \times 140 \mathrm{~mm})$ or $750 \times 750$ elements $(210 \mathrm{~mm} \times 210 \mathrm{~mm})$. Each tissue element can represent either excitable myocardial tissue or inexcitable post-infarction scar. Grids with more elements were used for modeling post-infarction scars with radius $r_{I S}>16.8 \mathrm{~mm}$.

Boundary conditions were formulated as the no flux through the boundaries:

$$
\vec{n} \nabla V=0
$$

where $\vec{n}$ is the normal to the boundary. Infarction scar elements were simulated as nonconducting inexcitable obstacles and considered as internal boundaries (no flux) for the myocardial elements.

To solve the problem (1)-(3), we used a finite-difference method with a 9-point stencil discretization scheme, as described in [24,25], with $0.28 \mathrm{~mm}$ for the spatial step and $0.02 \mathrm{~ms}$ for the time step. Rush-Larsen formalism [26] was used for TP06 gating variables integration.

\subsection{Representation of Post-Infarction Scar and Gray Zone}

Post-infarction scar (IS) was modeled as a non-conducting inexcitable obstacle: we imposed no-flux boundary conditions (3) at the boundary of this domain. In most of the simulations, we used a circular obstacle at the center of the tissue (black area in Figure 1A). It is surrounded by a gray zone (gray area in Figure 1A). For some simulations, we also use the model of the IS with a realistic shape from [27] (Figure 1B). Because the period of rotation around an obstacle with concavities is determined by the convex hull of the obstacle [14], we determined the perimeter of a convex hull for the obstacle in Figure 1B, which is $116.1 \mathrm{~mm}$.

We used a TP06 cellular ionic model [23] with referent parameters for every cell in the healthy myocardial tissue, and a model with modified parameters (Table 1) for every cell in the gray zone reflecting cellular remodeling in myocardial tissue around the scar. Modification of the TP06 ionic model for the description of cells in the gray zone was taken from [28] and is similar to the properties of gray zone cells from [21]. In particular, the maximal conductances of several ionic currents are changed against the reference values as specified in Table 1. The difference in the model parameters result in the difference in the action potential shape and duration between the cells from the gray zone and healthy myocardium (Figure 1B). The action potential duration is longer in the gray zone. The difference in the cellular activity between the border zone and the rest of the healthy tissue provides the functional heterogeneity in myocardial tissue around the scar. 
Table 1. Maximal conductivity $\left(G_{*}\right)$ of ionic currents in cellular model from the normal tissue (referent values) and gray zone (modified values).

\begin{tabular}{lcc}
\hline Current & Normal Tissue & Gray zone \\
\hline Fast Na+ current $\left(I_{N a}\right)$ & 14.838 & $12.6123\left(0.85 \times G_{N a}\right)$ \\
L-type $C a^{2+}$ current $\left(I_{C a L}\right)$ & $3.98 \times 10^{-5}$ & $3.03674 \times 10^{-5}\left(0.763 \times G_{C a L}\right)$ \\
Rapid delayed rectifier current $\left(I_{K r}\right)$ & 0.134 & $0.0938\left(0.7 \times G_{K r}\right)$ \\
Slow delayed rectifier current $\left(I_{K s}\right)$ & 0.27 & $0.054\left(0.2 \times G_{K s}\right)$ \\
Inward rectifier $\mathrm{K}^{+}$current $\left(I_{K 1}\right)$ & 5.405 & $1.621\left(0.3 \times G_{K 1}\right)$ \\
Transient outward current $\left(I_{t o}\right)$ & 0.29372972 & $0.029372972\left(0.1 \times G_{t o}\right)$ \\
\hline
\end{tabular}
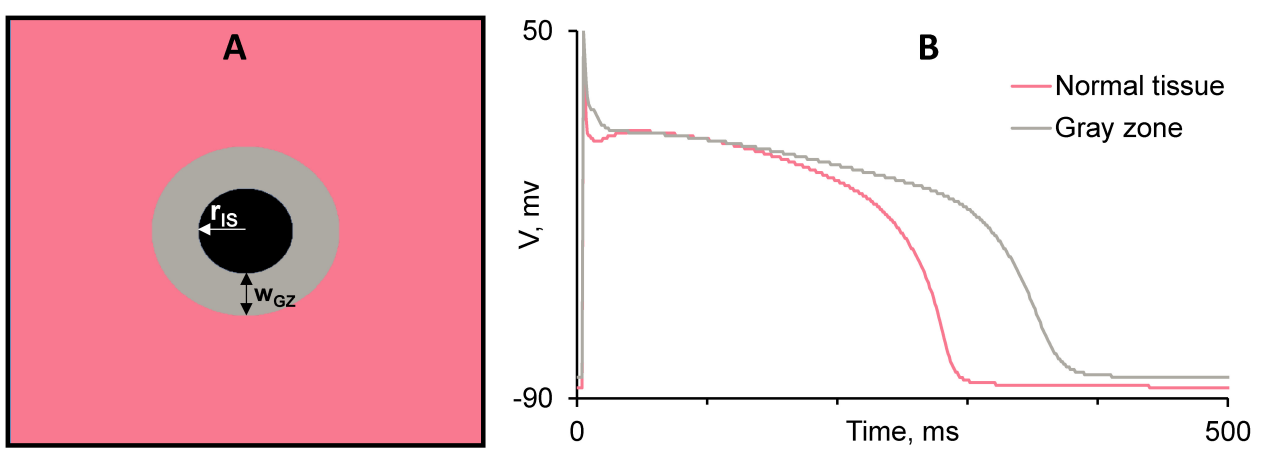

Normal (healthy) tissue

Gray zone

Infarction scar
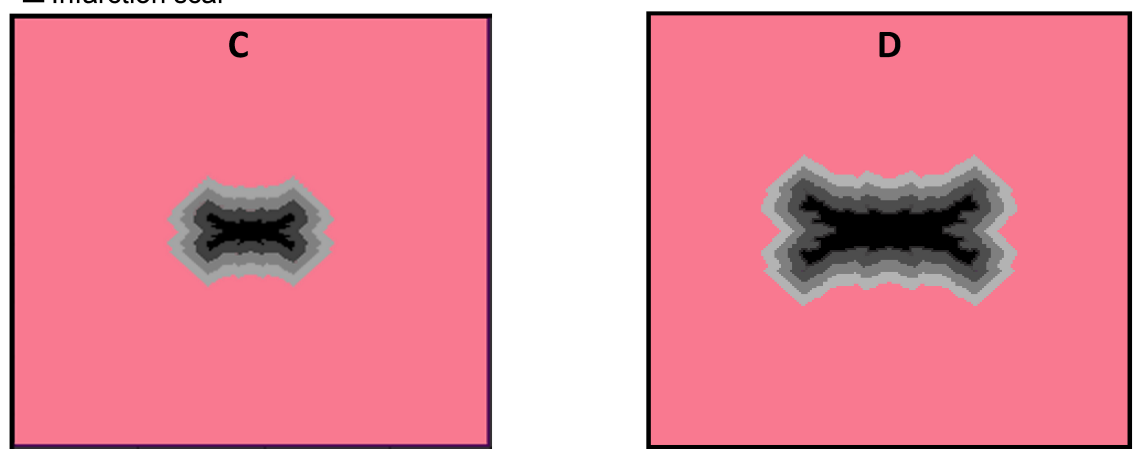

Figure 1. (A) Schematic representation of the geometry of cardiac tissue with the post-infarction scar. Normal tissue (pink), post-infarction scar (black) and gray zone around it (gray). (B) the shape of the action potential vs. time in cells of healthy myocardium (pink) and gray zone (gray). (C,D) Models of cardiac tissue with the post-infarction scar with a realistic shape. The gray zones of different sizes (shades of gray) are shown.

\subsection{Protocol of Numerical Experiments in 2D Tissue}

A rotating wave in a 2D myocardial tissue slab with a post-infarction scar and gray zone was initiated counterclockwise using a standard protocol S1-S2 (see Figure S1 in the Supplementary Materials for a graphical description of the protocol). After the wave rotation was stabilized, the period of rotation was determined as the time interval between the subsequent moments of wave arrival to a given point in the healthy tissue.

We varied the size of the scar from $r_{I S}=0$ to $r_{I S}=53.2 \mathrm{~mm}$ with a step of $2.8 \mathrm{~mm}$, and width of the gray zone from $w_{G Z}=0$ to $w_{G Z}=42 \mathrm{~mm}$, also with a step of $2.8 \mathrm{~mm}$ and analyzed the wave rotation pattern and determined the rotation period.

Note, in this study, we assume that the properties of cardiac cells in the gray zone and in normal tissue are spatially uniform and thus we use only one parameter set to describe the normal tissue and one parameter set to describe the gray zone. As a result, after the wave rotation was stabilized, we were obtaining only one value of the period of rotation for each geometry. Thus, a single model run per each pair of $\left(r_{I S}, w_{G Z}\right)$ was performed. 
All of the calculations were performed on a C program on clusters "URAN" (IMM, Ural Branch of RAS) and "IIP" of the Institute of Immunology and Physiology (Ekaterinburg). The program uses CUDA for GPU parallelization and was compiled with a Nvidia C Compiler "nvcc". The computational nodes have graphical cards Tesla K40m0.

\section{Results}

\subsection{Wave Rotation Regimes}

Varying the radius of a post-infarction scar $\left(r_{I S}\right)$ and the gray zone width $\left(w_{G Z}\right)$ in our 2D tissue models, we observed several qualitatively different spatial patterns of the wave rotation. Representative types of the wave patterns are shown in Figure 2.
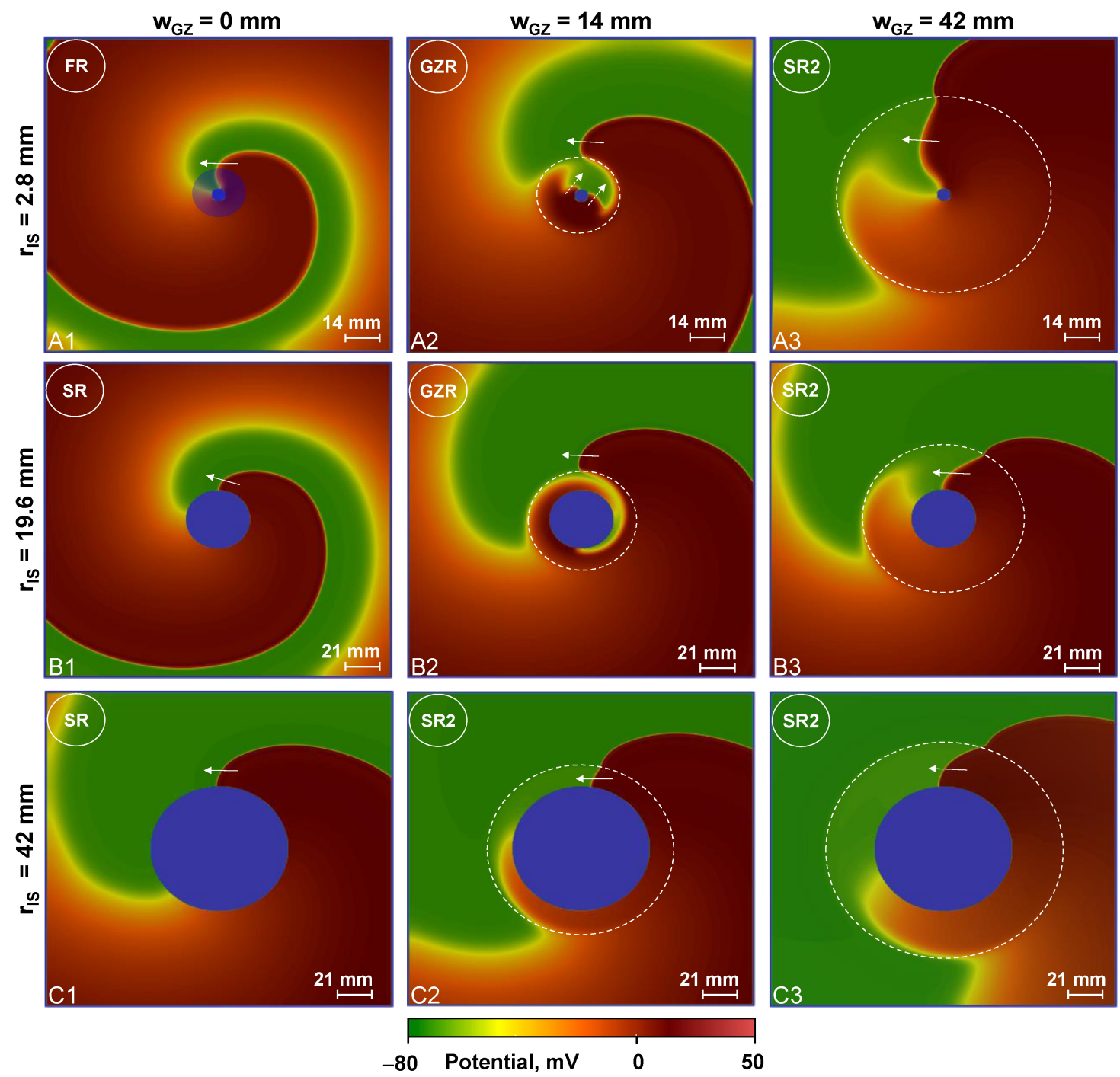

Figure 2. Examples of waves rotating around post-infarction scars of different sizes (top to bottom: $r_{I S}=2.8 \mathrm{~mm} ; 19.6 \mathrm{~mm}$; $42 \mathrm{~mm}$ ) with a gray zone of different sizes (left to right: $w_{G Z}=0 \mathrm{~mm} ; 14 \mathrm{~mm} ; 42 \mathrm{~mm}$ ). Four rotation regimes are shown: scar rotation (SR), functional rotation (FR, blue shading shows the effective obstacle around which the wave rotates), gray zone rotation (GZR) and scar rotation 2 (SR2). See the text for the description of the rotation regimes. Left column: typical view of the wave rotating around the scar in the absence of the gray zone $\left(w_{G Z}=0 \mathrm{~mm}\right)$. Waves rotate in two regimes: $F R$ (A1) and $S R$ (B1, C1). Middle column: waves rotate around the gray zone in a GZR (A2, B2) and SR2 (C2). Right column: $\left(w_{G Z}=42 \mathrm{~mm}\right) S R 2$ regime in all cases (A3-C3). Arrows show the direction of the wave rotation. Dashed arrows in A2 $\left(r_{I S}=19.6 \mathrm{~mm}, w_{G Z}=14 \mathrm{~mm}\right)$ show wave direction inside the gray zone. Dashed circles show the border of the gray zones. Movies of rotation of spiral waves in GZR and SR2 regimes can be found in the Supplementary Materials (movies S2 and S3). 
In the absence of the gray zone (Figure $2 \mathrm{~B} 1, \mathrm{C} 1, w_{\mathrm{GZ}}=0 \mathrm{~mm}$ ), the rotation occurs with the wavefront orthogonal to the boundary of the scar. Let us call this regime a scar rotation, as here the radius or rotation is determined by the radius of the scar. However, for a small scar in Figure 2A1, the situation is different. It looks as if the wave is rotating around an effective obstacle of a larger radius: the point where displacement in the normal direction coincides with the visible rotation around a center is located on a circle of a larger radius than the scar (blue shaded circle). Let us call such regime a functional rotation. That means that the radius of rotation here is determined by the wave itself and not by the obstacle.

For the gray zone of $w_{G Z}=14 \mathrm{~mm}$ (Figure 2A2,B2), the rotation occurs with the wavefront orthogonal to the boundary of the gray zone, as if it is non-excitable. Inside the gray zone, we see an area in red-orange colors indicating that spatial duration of the action potential here in prolonged. Let us call this regime a gray zone rotation, as here, the radius or rotation is determined by the radius of the gray zone. For the largest scar in Figure 2C2, the wavefront is attached and almost orthogonal to the scar and we observe scar rotation, however, as the wave here moves inside the gray zone, let us call this regime a scar rotation 2.

For the largest gray zones of $w_{\mathrm{GZ}}=42 \mathrm{~mm}$ (Figure $2 \mathrm{~B} 3, \mathrm{C} 3$ ), we see the scar rotation 2 regime, except for in Figure 2A3, where we see some features of functional rotation inside the gray zone.

Now let us analyze the period of rotation, which is one of the main characteristics of cardiac arrhythmia.

\subsection{Rotation Period Depending on Geometry of the Scar}

We plotted dependencies of the rotation period on the radius of the scar for four values of the gray zone width (Figure 3A) and dependencies of the rotation period on the width of the gray zone for four values of the scar radius (Figure 3B).

Let us consider Figure 3A first. The black line shows the rotation period vs. $r_{I S}$ in the absence of the gray zone $\left(w_{G Z}=0\right)$. We see that for small $r_{I S}\left(r_{I S}<14 \mathrm{~mm}\right)$ the period is almost constant and does not depend on the size of the scar, and then dependence becomes linear with a slope of 6.95. Similarly, in the case where the entire tissue has the properties of the gray zone, the dashed line $\left(w_{G Z}=\infty\right)$, we have a dependency with the region of almost a constant period and then monotonically increasing dependency approaching linearly with a slope of 5.45. (Here, the entire tissue has the properties of the gray zone.) We will use this line later to explain the mechanisms of transitions between different wave rotation regimes. For the gray zone with a width of $w_{G Z}=8.4 \mathrm{~mm}$ (the blue line), we have a similar dependency, with a linear slope $=6.32$, however, the transition to the linear part here starts for smaller infarct radius values of $r_{I S}>2.8 \mathrm{~mm}$. For the gray zone with $w_{G Z}=30.8$ and $36.4 \mathrm{~mm}$ (green and yellow lines, respectively), both lines approach the asymptotic dashed line at large scars. For a smaller scar radius, the dependencies are linear before the green and yellow lines approach the asymptotic dashed line. Their slopes here are 6.35 and 6.5 , respectively.

Now, let us consider the dependence of the wave rotation period on the width of the gray zone at constant $r_{I S}$ (Figure 3B). For all $r_{I S}$, we have similar dependencies, which at small $w_{G Z}$ are almost linearly increasing functions that for larger $w_{G Z}$ instantly switch to the almost horizontal straight lines. We also see that for larger scars, the switch to the plateau occurs at lower $w_{G Z}$. In particular, for a small scar of $r_{I S}=8.4 \mathrm{~mm}$, the plateau phase starts around $w_{G Z}=36.0 \mathrm{~mm}$, while for the largest scar of $r_{I S}=36.4 \mathrm{~mm}$, the plateau is approached at around $w_{G Z}=16.8 \mathrm{~mm}$. We also performed simulations for the cases of post-infarction scar with realistic shapes (Figure 3B, gray lines). To place points on the graph, we calculated the effective radius corresponding to the perimeter of the scar defined as described in the methods section. We see that periods of a wave rotating around a realistic scar also linearly depends on width of the gray zone and are almost identical to the periods of the corresponding scar of a circular shape. 

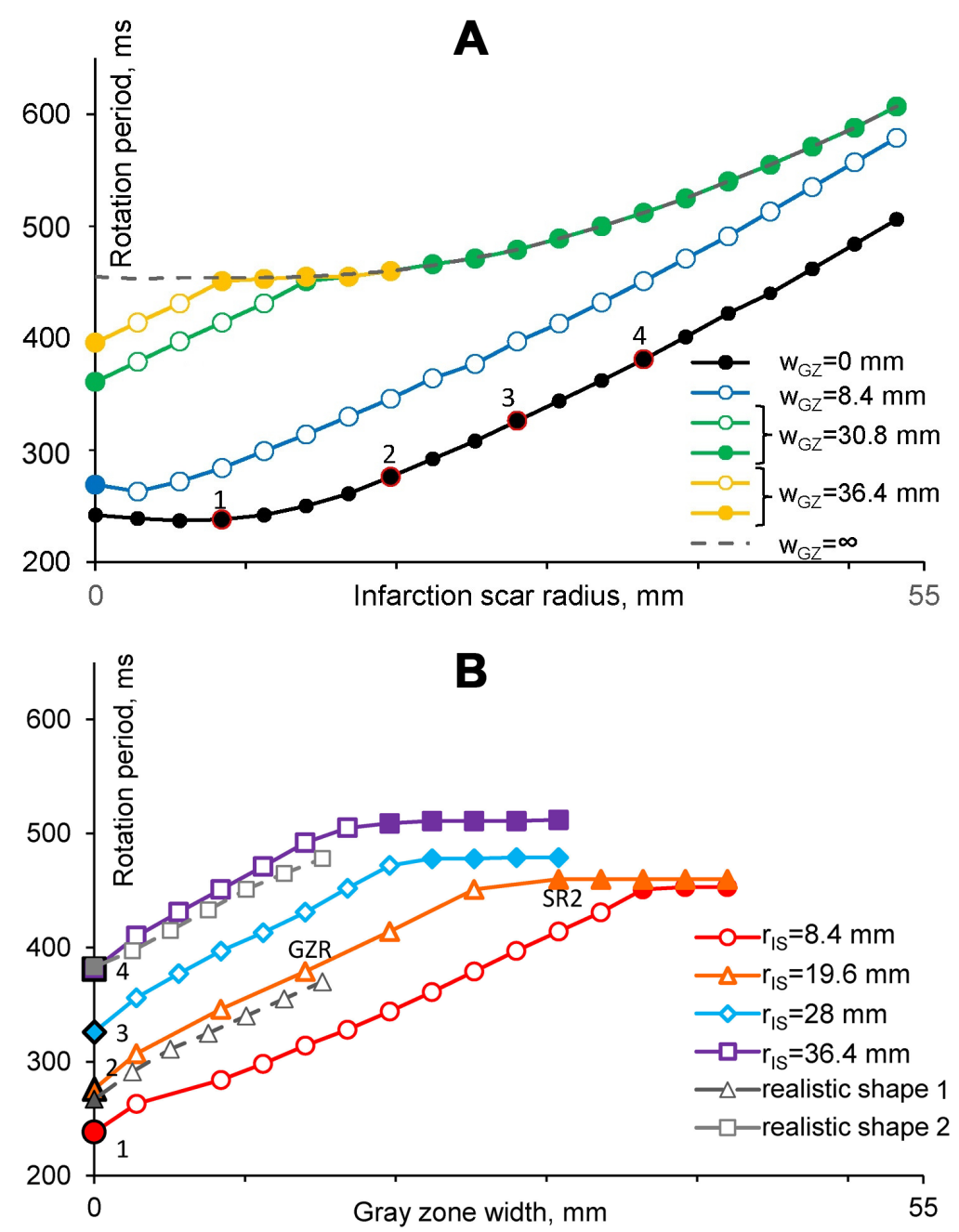

Figure 3. Dependence of the wave rotation period on the radius of the scar $\left(r_{I S}\right)$ and the width of the gray zone $\left(w_{G Z}\right)$. (A) Dependence of the wave rotation period on the radius of the scar with the gray zone of a different width. The black line shows cases in absence of the gray zone. The gray dashed line represents periods of waves rotating around a scar in tissue with properties of the gray zone $\left(w_{G Z}=\infty\right)$. (B) Dependence of the wave rotation period on the width of the gray zone in experiments with different radius of infarction scars. Red line shows a case with smaller radius of infarction scar $\left(r_{I S}=8.4 \mathrm{~mm}\right)$, purple line-case with bigger infarction scar $\left(r_{I S}=36.4 \mathrm{~mm}\right)$. Gray dashed lines show cases of infarction scars with a realistic shape. Scar perimeter is $116.1 \mathrm{~mm}$ for a scar with a realistic shape 1 (Figure 1C corresponds to the radius of ideal circular scar of $r_{I S}=18.5 \mathrm{~mm}$ ), and $233.5 \mathrm{~mm}$ for scar with a realistic shape 2 (Figure 1D corresponds to the radius of an ideal circular scar of $\left.r_{I S}=37.1 \mathrm{~mm}\right)$. Filled symbols $(\bullet)$ represent cases of the scar rotation regime. Open symbols (०) represent cases of the gray zone rotation regime. Marked dots (1)-(4) represent the same cases.

\subsection{Period and Regimes of Rotation}

Now, let us explain the observed dependencies and relate them to the rotation regimes defined in Section 3.1. Let us first consider the orange line in Figure 3B. Here the line starts at $w_{G Z}=0$ with the scar rotation regime and period of $276 \mathrm{~ms}$. We illustrate this wave pattern in Figure 2B1. When $w_{G Z}$ increases, the wave detaches from the obstacle and starts rotating around the gray zone in the gray zone rotation regime. We illustrate this wave pattern with the period of rotation of $379 \mathrm{~ms}$ in Figure 2B2. The reason for the onset of such a regime is the following. It turns out that for a wave, it is faster to rotate around the gray zone in the normal tissue than around a scar in the gray zone tissue, as the velocity of the wave there is smaller due to longer action potential in the gray zone. Indeed, we found 
that the period of rotation inside the gray zone for a scar of this size is $460 \mathrm{~ms}$. We can see it from the dashed line in Figure 3A for $r_{I S}=19.6 \mathrm{~mm}$. This period is longer than the period of $379 \mathrm{~ms}$ for rotation around the gray zone, and thus the wave chooses to rotate around the gray zone.

We can also explain the transition from the gray zone to the scar rotation regime if we further increase the size of the gray zone. Now, the period of rotation around the gray zone increases with its size, and for the gray zone of $w_{G Z}=30.8 \mathrm{~mm}$ it reaches a value of $460 \mathrm{~ms}$ (see dot marked SR2 in Figure 3B). For further increase in $w_{G Z}$, it will be faster for the wave to rotate around the scar than around the gray zone, and we observe the scar rotation 2 regime with a period of $460 \mathrm{~ms}$ (Figure 2, B3). Thus, a further increase in $w_{G Z}$ will not change the wave rotation regime and the period will remain $T=460 \mathrm{~ms}$, as we see in Figure 3B.

Note that as both the gray zone and scar rotations occur in the normal tissue, these regimes are closely related to each other. For example, in our case of $r_{I S}=19.6 \mathrm{~mm}$ and $w_{G Z}=14 \mathrm{~mm}$, the radius of the gray zone is $r_{I S}+w_{G Z}=33.6 \mathrm{~mm}$. From Figure 3A, we find that for a scar of $r_{I S}=33.6 \mathrm{~mm}$, the period of rotation is $362 \mathrm{~ms}$, which is indeed close to observed value of $379 \mathrm{~ms}$.

Now, let us consider the dependencies in Figure 3A. For $w_{G Z}=0$ (the black line), the linear part of the curve is associated with the scar rotation regime and increasing of the period of the wave is caused by increasing scar radius $r_{I S}$. However, the flat part of the dependency for small $r_{I S}$ is associated with the functional rotation regime. Indeed, for small $r_{I S}$, the rotation effectively occurs around a circle with the radius larger than the size of the scar, and thus the size of the scar does not affect the rotation period. An example of such an effective rotation circle is shown in Figure 2A1 by shaded blue. Similar wave patterns of the functional and scar rotation regimes are associated with the dashed curve $\left(w_{G Z}=\infty\right)$. However, here we obviously have the scar rotation 2 regime for large scars and the functional rotation regimes for small scars as the wave here propagates in the tissue with the properties of the gray zone.

For the gray zone with a large width of $w_{G Z}=30.8 \mathrm{~mm}$ (see green line in Figure 3A) and large scars $r_{I S}>=14$, the curve coincides with the dashed line corresponding to the scar rotation 2 regime. However, for $r_{I S}<14 \mathrm{~mm}$, we have the gray zone rotation regime as the rotation around the gray zone, which is faster than scar rotation 2 . For the gray zone with the largest width of $w_{G Z}=36.4$ (yellow line in Figure 3A), we have scar rotation 2, except smaller scars of $r_{I S}<8.4 \mathrm{~mm}$. Here, we have again the gray zone rotation. Compared to the green curve, the scar rotation 2 here occurs at the smaller scar radius. This is also logical, as for larger $w_{G Z}$, the corresponding curve is closer to its asymptotic dashed curve.

Finally, let us consider the factors which determine the slopes of the dependencies of the period of rotation on the scar geometry parameters.

\subsection{Factors Affecting the Period of Rotation}

To determine the factors responsible for the observed dependency of the period on the size of the scar and the gray zone, let us consider the following simple equation. If we assume that the velocity of wave $(v)$ at the boundary of the obstacle is constant and the perimeter of the obstacle is $L$, then the period $T$ is given by: $T=L / v$, or $L=T * v$. In turn, the velocity of the wave depends on the period of excitation $T$ (conduction velocity $(\mathrm{CV})$ restitution) and the curvature of the wave front [29,30]. If we neglect the dependency of the velocity on the curvature, we get: $L=T * v(T)$. Thus, the slope of the dependency of the period on the obstacle size (shown in Figure 3A) can be found from the following simple calculation:

$$
\begin{gathered}
\frac{d L}{d T}=\frac{d(T * v(T))}{d T}=v(T)+T * \frac{d v}{d T} \\
\frac{d T}{d L}=\frac{1}{v(T)+T * d v / d T}
\end{gathered}
$$

where $d v / d T$ is the slope of the CV restitution curve. 
Thus, we see that the slope of the dependency of wave period $T$ on the obstacle perimeter $L$ is determined not only by the velocity $v$ itself but also by the slope of the $C V$ restitution curve.

We computed CV restitution curves for the normal tissue and tissue with properties of the gray zone (Figure 4). We see that both curves are monotonically increasing functions $v(T)$ approaching some saturated values $\left(v_{N T}\right.$ for healthy tissue, and $v_{G Z}$ for gray zone tissue). Thus, for large pacing periods, $d v / d T$ approaches a zero value, and according to the formulae (4), the slope $d T / d L$ would approach the value $1 / v_{N T}$ or $1 / v_{G Z}$, respectively. However, such saturation occurs for rather long pacing periods, which are mostly outside of the physiologically relevant values. In our case, the rotation periods are in the range of $250<T<600$, where $v$ strongly depends on $T$ for both the $C V$ restitution curves. Let us estimate which terms in Equation (4) primarily determine the slope of the dependencies in Figure $3 \mathrm{~A}$, and explain why the curves with larger a gray zone have smaller slopes than the curve without the gray zone. Let us use period $T=500$, which is inside the region of linear dependency in Figure 3A, for both curves: with and without the gray zone. For the case of a scar without the gray zone (the black curve), at $T=500 \mathrm{~ms}$ we find that $v=0.678$, and $d v / d T=0.0003$ is derived from the black curve in Figure 4. We use it as, in this case, the wave rotates around the scar surrounded by the normal tissue. Thus, in Equation (4), the second term $T * d v / d T=0.15$ is substantially smaller than the first term $v$. For a large gray zone (green curve in Figure 3A), the rotation at $T=500 \mathrm{~ms}$ occurs inside the gray zone, thus, we need to use the $\mathrm{CV}$ restitution curve for the border zone tissue (the gray curve in Figure 4). Similarly, we find that for $T=500 \mathrm{~ms} v=0.46$, and $d v / d T=0.00195$, thus, in Equation (4) $T * d v / d T=0.979$ is higher than $v$. Thus, in this case, we see that now the second term $T * d v / d T$ determines the slope of the dependency. Thus, we can conclude that the lower slope of the dependency of $\mathrm{T}$ on $\mathrm{L}$ in the presence of the gray zone is a result of CV restitution slope $(\mathrm{dv} / \mathrm{dT})$, where a larger derivative occurs due to 'shift' of the $\mathrm{CV}$ restitution curve to the longer periods because of tissue remodeling in the gray zone (Figure 4). Note, however, that linear dependencies which we see in Figure 3A for large radii are actually just a coincidence for specific values of parameters. The slopes will further change and for very large values of $r$, they approach the values of $1 / v_{N T}$ and $1 / v_{G Z}$ correspondingly. At that stage, the slope of the dashed line in Figure $3 \mathrm{~A}$ will be larger than that of the black line. However, it occurs far beyond the physiologically relevant values for spiral waves.

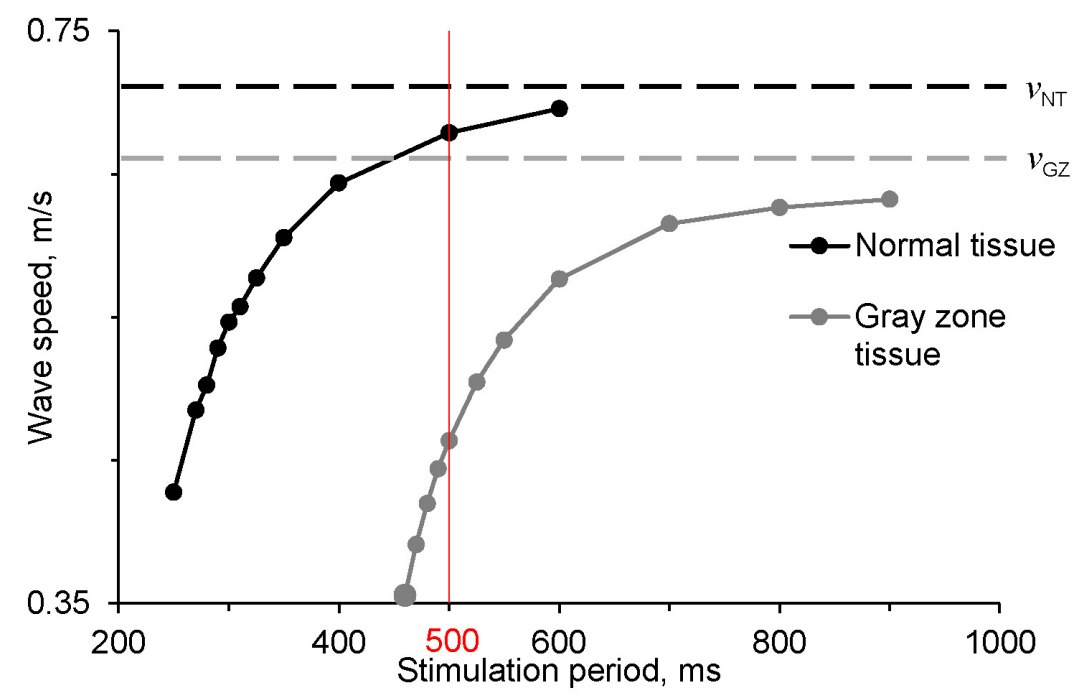

Figure 4. Dependence of the wave speed on the activation period of the wave (restitution curve). Wave speed in normal tissue (black) and tissue with properties of the gray zone (gray) are shown. Black and gray dashed lines show the maximum speed of waves in normal tissue $\left(v_{N T}=0.71 \mathrm{~m} / \mathrm{s}\right)$ and in the gray zone tissue $\left(v_{G Z}=0.66 \mathrm{~m} / \mathrm{s}\right)$, respectively. 


\subsection{Additional Rotation Regimes}

In addition to the regimes discussed above, for a few parameter values, we also observed more complex rotation regimes. All of these regimes occur for small scars and can be distinguished into two types: active and passive. Figure 5A shows an example of a passive regime, where we periodically see a breakthrough of excitation into the gray zone, however, they do not disturb the leading edge wave rotation. Figure $5 B$ shows an example of an active regime. Here, the wave comes outside the gray zone, actively interacts with the leading edge of excitation and changes the trajectory of the wave rotation. As a result, we observe a non-monotonical dependency of the period on the size of the scar. However, the active regime was not frequently observed, we saw it only in 3 cases (out of 360) with the perimeter of the gray zone neing $52.7 \mathrm{~mm}\left(r_{I S}=0 \mathrm{~mm}, w_{G Z}=8.4 \mathrm{~mm} ; r_{I S}=2.8 \mathrm{~mm}\right.$, $w_{G Z}=5.6 \mathrm{~mm} ; r_{I S}=5.6 \mathrm{~mm}, w_{G Z}=2.8 \mathrm{~mm}$ ), thus, they may have limited importance for the overall dynamics.

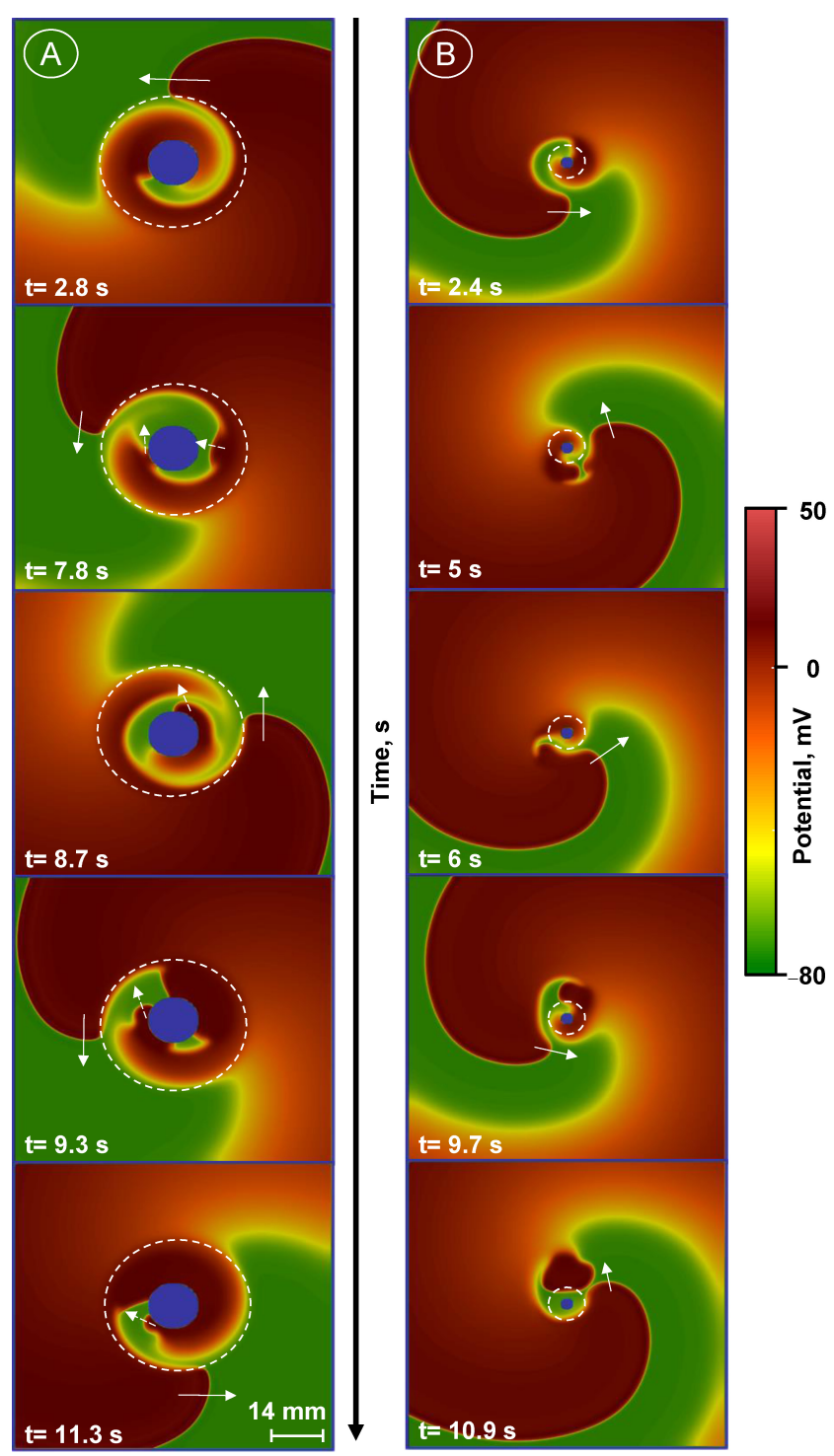

Figure 5. Examples of waves with dynamical instabilities. Border of the gray zone is depicted by the dashed line. Arrows show the direction of the wave rotation. Dashed arrows show wave direction inside the gray zone. (A) A passive regime. The breakthrough waves marked by dashed arrows do not exit the gray zone $\left(r_{I S}=11.2 \mathrm{~mm}, w_{G Z}=25.2 \mathrm{~mm}\right)$. (B) An active regime. The breakthrough waves exit the gray zone and force the wave to rotate at some distance from the border of the gray zone $\left(r_{I S}=2.8 \mathrm{~mm}, w_{G Z}=5.6 \mathrm{~mm}\right.$.) An illustration of such dynamics can be found in the Supplementary Materials (movies S4 and S5). 


\section{Discussion}

In this paper, we perform a detailed study of the dynamics of the waves rotating around a region, which generically represents a post-infarction scar and includes a compact inexcitable obstacle surrounded by a gray zone with modified properties of cardiac cells as compared to the rest of cardiac tissue. We found that all of the observed dynamics can be subdivided into several main classes. These classes naturally occur if we combine properties of a wave rotating around an inexcitable obstacle and the effects of tissue heterogeneity on the wave propagation.

In the absence of tissue heterogeneity, the period of the wave rotating around an obstacle is mainly determined by the velocity of the wave and the perimeter of the obstacle [14]. This is a scar rotation regime, which was observed in previous studies. However, compared to earlier studies [14], we show that one needs to take into account the dependency of the velocity on the period ( $\mathrm{CV}$ restitution), and explain its dependence on the size of the scar. Another observed regime of functional rotation was found in early studies in the reaction diffusion models [15]. It was shown that for small obstacles, the period of rotation does not decrease anymore, wave detaches from the obstacle and rotates around a circle which exceeds the obstacle size. In [15], it was also shown that detachment from the obstacle is associated with a large increase in the period of rotation. We did not observe such a period increase in our study. This probably occurred because in [15], a two variable model with lower excitability was used. In our study, we used an ionic model with high excitability, and thus the boundary effects of the obstacle on the wave propagation velocity in our case are less pronounced.

In the presence of the gray zone, the situation is more complex and, as far as we are aware, was not yet analyzed in details. Our study shows that here we can observe additional rotation regimes, which we call gray zone rotation and scar rotation 2 regimes. We show that dependencies of the period on the size of the scar and the gray zone are bound by two asymptotic solutions for homogeneous tissue: rotation of the wave around a scar in normal tissue and rotation of the wave around a scar in tissue with properties of the gray zone. As we show in Figure 3A, all of the curves for different sizes of the gray zone are located between these two asymptotic lines and the slopes of these lines are bound from above and below the slopes of all of the other curves. In addition, we found a new regime, gray zone rotation. In that case, the wave rotates as if the compact scar coincides with the gray zone. In the gray zone rotation regime, the period of rotation depends on the perimeter of the gray zone, and not on the size of the scar. We illustrate it in Figure 6, where we show the dependence of the period on the perimeter of the gray zone for various sizes of the scar. We see that all of the curves in a certain range of the parameters almost coincide, which, one more time, confirms that, here, the perimeter of the gray zone, and not the scar size, determines the period of rotation. However, we also see that these curves with the gray zone are a little above the dashed line, representing the case of pure scar rotation. It indicates that the leading edge of rotation for the gray zone rotation regime is a little outsize the boundary of the gray zone, which maybe be a result of the electrotonic effects [31].

An important rule for transition between the gray zone and scar rotation regimes is the minimal period principle proposed for such a situation in this paper. In fact, if the rotation in normal tissue along the gray zone is faster than the rotation around the scar in the gray zone, then the gray zone regime will occur. Such a transition is clearly seen in Figure $3 \mathrm{~A}, \mathrm{~B}$ where we see a change from one type of dependency to another one. Note, this also implies that transitions between the regimes strongly depend on the relation of the velocity of the wave inside and outside the gray zone. Here, we considered only one type of cellular heterogeneity based on the data reported in [21,28], where action potentials are longer in the gray zone than in the normal cardiac tissue. However, in [32], it was shows that remodeling in the gray zone can also result in different changes in the properties of cardiac tissue and can even result in shortening of the action potential. It would be interesting to study similar regimes of wave rotation for different properties of cardiac tissue in the gray zone. In addition, the gray zone can also include fibrosis and a reduced 
number of gap junctions. It also can decrease the propagation velocity and thus make the gray zone rotation regime more essential.

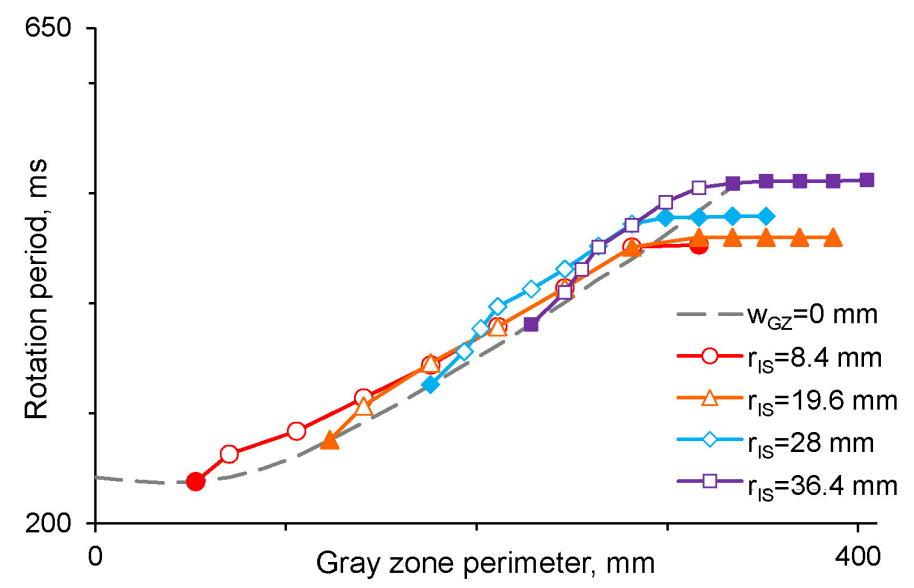

Figure 6. Dependence of the wave rotation period on the perimeter of the gray zone for different radii of an infarction scar. Red line shows the case with smallest radius of scar core, purple line-case with the biggest scar core. The dashed line connects points where the gray zone is absent $\left(w_{G Z}=0 \mathrm{~mm}\right)$.

The dynamics of rotating waves around an obstacle was studied in [17], where a general theory for the dynamics and instability of pinned spirals was developed. In particular, it was shown that the destabilization is better understood by the implementation of a mapping rule and dimension reduction.

Moreover, the transition between scar and functional rotation when the radius of the obstacle decreases was studied in [33]. There, a sequence of transitions, from periodic motion to a modulated period-2 rhythm, and then to spiral wave breakup [34,35], were observed. It would be interesting to study how such dynamical instabilities will manifest themselves in the presence of tissue heterogeneity around a scar, as considered in our paper.

It would be also interesting to study such rotational activity in anatomical models of the heart $[21,36]$ and also include additional types of regional cellular heterogenety in the normal tissue as considered in $[37,38]$.

In our paper, the properties of the gray zone were represented via changes in cell-level models. However, there are also changes in conductivity between the cells. In [39], it was shown that in the gray zone, the muscle fibers were widely separated and disoriented by the connective tissue. In [40], it was further shown that there was decreased side-toside coupling between myocytes in the gray zone, which results in increased anisotropy because of decreased transverse conduction velocity. A direct way to account for this change is to modify the diffusion coefficient in the term $D \nabla^{2} V$ in Equation (1) by reducing its eigenvalue, accounting for the transverse propagation [21]. In our paper, we limited our study to the isotropic case, and thus, adding such change is beyond the scope of this research. However, in the presence of anisotropy, reduction in the transversal condition will decrease the overall velocity of wave propagation and thus will be an additional factor affecting the period of wave rotation. It will be interesting to extend our studies to the case of anisotropic tissue and investigate the effects of decreasing of the transversal velocity on the period of the arrhythmia. Form our analysis presented in this paper, especially from the minimal period principle, we expect that a further decrease in the conduction velocity will make rotation in normal tissue more preferable, and thus will further increase the range of parameters where the gray zone rotation will be observed.

In this paper, we apply mathematical methods to study the factors which determine the period of arrhythmia in a generic model of cardiac tissue. However, the applications of mathematical methods in cardiac research are much more wide-ranging, for example, for the analysis of population studies [41]. Moreover, currently in vitro cardiac models from stem cells are of great interest $[42,43]$. Such a system can also be studied using 
corresponding mathematical models [44]. However, the models for such systems should be based on the standards for recording, annotating and reporting experimental data reviewed in [45].

Here, we study only one mechanism of arrhythmias associated with rotating waves. Another important mechanisms is triggered activity, which occurs close to point of Hopf bifurcation [46] and is associated with the onset of focal sources of excitation due to early afterdepolarizations. Such conditions normally occur due to pathological elongation of the action potential [47]. Because elongation of action potential occurs in the gray zone [48], it would be interesting to study the manifestation of arrhythmias based on the mechanisms of early afterdepoarizations, which can occur in the gray zone close to the infarction scar.

In conclusion, we found that the main dynamics of wave rotation around an obstacle surrounded by heterogeneous ventricular myocardial tissue include functional, scar and a newly identified gray zone rotation regimes. The transition between the regimes can be understood and predicted from the proposed minimal period principle. The slopes of dependencies of the period on the size of the obstacle can be quantitatively calculated from the conduction velocity restitution.

Supplementary Materials: The following are available at https://www.mdpi.com/article/10.339 0/math9233090/s1, Figure S1: Graphical description of the protocol S1-S2. Video S2: Example of wave rotating in gray zone rotation regime. Infarction scar size: $r_{I S}=19.6 \mathrm{~mm}$. Gray zone width $w_{G Z}=14 \mathrm{~mm}$. Video S3: Example of wave rotating in scar rotation 2 regime. Infarction scar size: $r_{I S}=19.6 \mathrm{~mm}$. Gray zone width $w_{G Z}=42 \mathrm{~mm}$. Video S4: Example of the wave with passive dynamic instability in gray zone. Infarction scar size: $r_{I S}=11.2 \mathrm{~mm}$. Gray zone width $w_{G Z}=22.4 \mathrm{~mm}$. Video S5: Example of the wave with active dynamic instability in gray zone. Infarction scar size: $r_{I S}=2.8 \mathrm{~mm}$. Gray zone width $w_{G Z}=5.6 \mathrm{~mm}$.

Author Contributions: Conceptualization, A.V.P., D.M. and O.S.; formal analysis, P.K., D.M.; investigation, P.K.; methodology, A.V.P. and P.K.; software P.K., A.D. and D.M.; supervision, A.P. and O.S.; visualization, P.K. and A.D.; writing-original draft preparation, P.K., A.V.P. and O.S.; writingreview and editing, P.K., A.P. and O.S. All authors have read and agreed to the published version of the manuscript.

Funding: A.V.P., P.K., D.M., A.D. and O.S. were funded by the Russian Foundation for Basic Research (\#18-29-13008). The work of P.K., D.M., A.D. and O.S. was carried out within the framework of the IIF UrB RAS theme No AAAA-A21-121012090093-0.

Data Availability Statement: Data related to this study can be provided by the corresponding authors on request.

Acknowledgments: We are thankful to A.M. Pertsov for a valuable discussion.

Conflicts of Interest: The authors declare no conflict of interest.

\section{Abbreviations}

The following abbreviations are used in this manuscript:

$\begin{array}{ll}\text { CV } & \text { Conduction velocity } \\ \text { FR } & \text { Functional rotation } \\ \text { GZ } & \text { Gray zone } \\ \text { GZR } & \text { Gray zone rotation } \\ \text { IS } & \text { Post-infarction scar } \\ \text { NT } & \text { Normal tissue } \\ \text { SR } & \text { Scar zone rotation } \\ \text { SR2 } & \text { Scar rotation } 2\end{array}$

\section{References}

1. Cross, M.C.; Hohenberg, P.C. Pattern formation outside of equilibrium. Rev. Mod. Phys. 1993, 65, 851. [CrossRef]

2. Winfree, A.T.; Strogatz, S.H. Organizing centres for three-dimensional chemical waves. Nature 1984, 311, 611-615. [CrossRef] 
3. Pertsov, A.M.; Aliev, R.R.; Krinsky, V.I. Three-dimensional twisted vortices in an excitable chemical medium. Nature 1990, 345, 419-421. [CrossRef]

4. Gorelova, N.A.; Bureš, J. Spiral waves of spreading depression in the isolated chicken retina. J. Neurobiol. 1983, 14, 353-363. [CrossRef]

5. Siegert, F.; Weijer, C.J. Three-dimensional scroll waves organize Dictyostelium slugs. Proc. Natl. Acad. Sci. USA 1992, 89, 6433-6437. [CrossRef]

6. Winfree, A.T. Spiral waves of chemical activity. Science 1972, 175, 634-636. [CrossRef]

7. Davidenko, J.M.; Pertsov, A.V.; Salomonsz, R.; Baxter, W.; Jalife, J. Stationary and drifting spiral waves of excitation in isolated cardiac muscle. Nature 1992, 355, 349-351. [CrossRef]

8. Jalife, J. Ventricular fibrillation: Mechanisms of initiation and maintenance. Annu. Rev. Physiol. 2000, 62, 25-50. [CrossRef]

9. Karma, A. Physics of cardiac arrhythmogenesis. Annu. Rev. Condens. Matter Phys. 2013, 4, 313-337. [CrossRef]

10. Bernus, O.; Zemlin, C.W.; Zaritsky, R.M.; Mironov, S.F.; Pertsov, A.M. Alternating conduction in the ischaemic border zone as precursor of reentrant arrhythmias: A simulation study. EP Eur. 2005, 7, S93-S104. [CrossRef]

11. Lopez, E.M.; Malhotra, R. Ventricular tachycardia in structural heart disease. J. Innov. Card. Rhythm Manag. 2019, 10, 3762. [CrossRef] [PubMed]

12. Stevenson, W.G. Ventricular scars and ventricular tachycardia. Trans. Am. Clin. Climatol. Assoc. 2009, 120, 403. [PubMed]

13. Connolly, A.J.; Bishop, M.J. Computational representations of myocardial infarct scars and implications for arrhythmogenesis. Clin. Med. Insights: Cardiol. 2016, 10, S39708. [CrossRef] [PubMed]

14. Weiner, N.; Rosenblunth, A. The mathematical formulation of the problem of conduction of impulses in a network of connected excitable elements specifically in cardiac muscle. Arch. Inst. Cardiol. Mex. 1946, 16, 205-265.

15. Pertsov, A.M.; Ermakova, E.A.; Panfilov, A.V. Rotating spiral waves in a modified Fitz-Hugh-Nagumo model. Phys. D Nonlinear Phenom. 1984, 14, 117-124. [CrossRef]

16. Vinson, M.; Pertsov, A.; Jalife, J. Anchoring of vortex filaments in 3D excitable media. Phys. D Nonlinear Phenom. 1994, 72, 119-134. [CrossRef]

17. Gao, X.; Feng, X.; Li, T.C.; Qu, S.; Wang, X.; Zhang, H. Dynamics of spiral waves rotating around an obstacle and the existence of a minimal obstacle. Phys. Rev. E 2017, 95, 052218. [CrossRef]

18. Balaban, G.; Halliday, B.P.; Bai, W.; Porter, B.; Malvuccio, C.; Lamata, P.; Rinaldi, C.A.; Plank, G.; Rueckert, D.; Prasad, S.K. Scar shape analysis and simulated electrical instabilities in a non-ischemic dilated cardiomyopathy patient cohort. PLoS Comput. Biol. 2019, 15, e1007421. [CrossRef]

19. Baba, S.; Dun, W.; Cabo, C.; Boyden, P.A. Remodeling in cells from different regions of the reentrant circuit during ventricular tachycardia. Circulation 2005, 112, 2386-2396. [CrossRef]

20. Schmidt, A.; Azevedo, C.F.; Cheng, A.; Gupta, S.N.; Bluemke, D.A.; Foo, T.K.; Gerstenblith, G.; Weiss, R.G.; Marbán, E.; Tomaselli, G.F. Infarct tissue heterogeneity by magnetic resonance imaging identifies enhanced cardiac arrhythmia susceptibility in patients with left ventricular dysfunction. Circulation 2007, 115, 2006-2014. [CrossRef]

21. Arevalo, H.J.; Vadakkumpadan, F.; Guallar, E.; Jebb, A.; Malamas, P.; Wu, K.C.; Trayanova, N.A. Arrhythmia risk stratification of patients after myocardial infarction using personalized heart models. Nat. Commun. 2016, 7, 1-8. [CrossRef]

22. Panfilov, A.; Holden, A. Computer simulation of re-entry sources in myocardium in two and three dimensions. J. Theor. Biol. 1993, 161, 271-285. [CrossRef]

23. ten Tusscher, K.; Panfilov, A.V. Alternans and spiral breakup in a human ventricular tissue model. Am. J. Physiol.-Heart Circ. Physiol. 2006, 291, H1088-H1100. [CrossRef]

24. Ten Tusscher, K.H.W.J.; Panfilov, A.V. Modelling of the ventricular conduction system. Prog. Biophys. Mol. Biol. 2008, 96, 152-170. [CrossRef]

25. De Coster, T. The Role of Adipose Tissue in Arrhythmogenesis: An In Silico Study. Ph.D. Thesis, Ghent University, Ghent, Belgium, 2019.

26. Rush, S.; Larsen, H. A practical algorithm for solving dynamic membrane equations. IEEE Trans. Biomed. Eng. 1978, 4, 389-392. [CrossRef]

27. Majumder, R.; Pandit, R.; Panfilov, A.V. Turbulent electrical activity at sharp-edged inexcitable obstacles in a model for human cardiac tissue. Am. J. Physiol.-Heart Circ. Physiol. 2014, 307, H1024-H1035. [CrossRef]

28. Decker, K.F.; Rudy, Y. Ionic mechanisms of electrophysiological heterogeneity and conduction block in the infarct border zone Am. J. Physiol.-Heart Circ. Physiol. 2010, 299, H1588-H1597. [CrossRef]

29. Zykov, V.S. Analytic evaluation of the relationship between the speed of a wave of excitation in a two-dimensional excitable medium and the curvature of its front. Biofizika 1980, 25, 888-892.

30. Cabo, C.; Pertsov, A.M.; Baxter, W.T.; Davidenko, J.M.; Gray, R.A.; Jalife, J. Wave-front curvature as a cause of slow conduction and block in isolated cardiac muscle. Circ. Res. 1994, 75, 1014-1028. [CrossRef]

31. Defauw, A.; Kazbanov, I.V.; Dierckx, H.; Dawyndt, P.; Panfilov, A.V. Action potential duration heterogeneity of cardiac tissue can be evaluated from cell properties using Gaussian Green's function approach. PLoS ONE 2013, 8, e79607. [CrossRef]

32. Mendonca Costa, C.; Plank, G.; Rinaldi, C.A.; Niederer, S.A.; Bishop, M.J. Modeling the electrophysiological properties of the infarct border zone. Front. Physiol. 2018, 9, 356. [CrossRef] 
33. Xie, F.; Qu, Z.; Garfinkel, A. Dynamics of reentry around a circular obstacle in cardiac tissue. Phys. Rev. E 1998, 58, 6355. [CrossRef]

34. Panfilov, A.V.; Holden, A.V. Self-generation of turbulent vortices in a two-dimensional model of cardiac tissue. Phys. Lett. A 1990, 151, 23-26. [CrossRef]

35. Panfilov, A.V.; Holden, A.V. Spatiotemporal irregularity in a two-dimensional model of cardiac tissue. Int. J. Bifurc. Chaos 1991, 1, 219-225. [CrossRef]

36. Panfilov, A.; Keener, J.P. Re-entry in an anatomical model of the heart. Chaos Solitons Fractals 1995, 5, 681-689. [CrossRef]

37. Keldermann, R.H.; ten Tusscher, K.H.; Nash, M.P.; Hren, R.; Taggart, P.; Panfilov, A.V. Effect of heterogeneous APD restitution on VF organization in a model of the human ventricles. Am. J. Physiol.-Heart Circ. Physiol. 2008, 294, H764-H774. [CrossRef]

38. Keldermann, R.H.; Ten Tusscher, K.H.W.J.; Nash, M.P.; Bradley, C.P.; Hren, R.; Taggart, P.; Panfilov, A.V. A computational study of mother rotor VF in the human ventricles. Am. J. Physiol.-Heart Circ. Physiol. 2009, 296, H370-H379. [CrossRef] [PubMed]

39. Ursell, P.C.; Gardner, P.I.; Albala, A.; Fenoglio, J.J., Jr.; Wit, A.L. Structural and electrophysiological changes in the epicardial border zone of canine myocardial infarcts during infarct healing. Circ. Res. 1985, 56, 436-451. [CrossRef] [PubMed]

40. Yao, J.A.; Hussain, W.; Patel, P.; Peters, N.S.; Boyden, P.A.; Wit, A.L. Remodeling of gap junctional channel function in epicardial border zone of healing canine infarcts. Circ. Res. 2003, 92, 437-443. [CrossRef] [PubMed]

41. Sim, A.; Mukherjee, S. Potential Low Energy Availability (LEA) risk amongst amateur and recreational athletes in Singapore. Phys. Act. Health 2021, 5, 166-177. [CrossRef]

42. Smit, T.; Schickel, E.; Azimzadeh, O.; von Toerne, C.; Rauh, O.; Ritter, S.; Durante, M.; Schroeder, I.S. A Human 3D Cardiomyocyte Risk Model to Study the Cardiotoxic Influence of X-rays and Other Noxae in Adults. Cells 2021, 10, 2608. [CrossRef]

43. Kerr, C.M.; Richards, D.; Menick, D.R.; Deleon-Pennell, K.Y.; Mei, Y. Multicellular Human Cardiac Organoids Transcriptomically Model Distinct Tissue-Level Features of Adult Myocardium. Int. J. Mol. Sci. 2021, 22, 8482. [CrossRef]

44. Paci, M.; Koivumäki, J.T.; Lu, H.R.; Gallacher, D.J.; Passini, E.; Rodriguez, B. Comparison of the simulated response of three in silico human stem cell-derived cardiomyocytes models and in vitro data under 15 drug actions. Front. Pharmacol. 2021, 12,604713 .

45. Quinn, T.A.; Granite, S.; Allessie, M.A.; Antzelevitch, C.; Bollensdorff, C.; Bub, G.; Burton, R.A.B.; Cerbai, E.; Chen, P.S.; Delmar, M. Minimum Information about a Cardiac Electrophysiology Experiment (MICEE): Standardised reporting for model reproducibility, interoperability, and data sharing. Prog. Biophys. Mol. Biol. 2011, 107, 4-10. [CrossRef]

46. Tran, D.X.; Sato, D.; Yochelis, A.; Weiss, J.N.; Garfinkel, A.; Qu, Z. Bifurcation and chaos in a model of cardiac early afterdepolarizations. Phys. Rev. Lett. 2009, 102, 258103. [CrossRef]

47. Vandersickel, N.; Kazbanov, I.V.; Nuitermans, A.; Weise, L.D.; Pandit, R.; Panfilov, A.V. A study of early afterdepolarizations in a model for human ventricular tissue. PLoS ONE 2014, 9, e84595.

48. Jie, X.; Gurev, V.; Trayanova, N. Mechanisms of mechanically induced spontaneous arrhythmias in acute regional ischemia. Circ. Res. 2010, 106, 185-192. [CrossRef] 